'FM Moliterno, 'EO Veiga, ${ }^{2} \mathrm{NV}$ Moliterno, ${ }^{2} \mathrm{~A}$ Veiga, ${ }^{2} \mathrm{SS}$ Cordeiro, ${ }^{1} \mathrm{SA}$ Nogueira. 'Paediatrics; ${ }^{2}$ Faculdade de Medicina de Petrópolis, Petrópolis, Brazil

Background and Aims Pneumonia ranks second in the table of infant mortality in children under 5 years. The incidence of pneumonia is $5-10$ times greater in developing countries. This study aimed to identify cases of pneumonia admitted to the Pediatrics Ward of the Hospital Alcides Carneiro, Rio de Janeiro, Brazil, from February to December 2008, which evolved satisfactorily using crystalline penicillin as monotherapy.

Methods Documentary analysis of the children diagnosed with pneumonia. The informed consent and informed about the objectives and procedures of the study was obtained from their legal guardians. Variables: age, sex, diagnosis, length of hospitalization, medication use, hospitalizations and outpatient medical care routine. We excluded children aged less than 61 days and those with chronic diseases.

Results Of 946 children admitted, 147 were diagnosed with pneumonia, outlining our study group. Ages ranged from 3 months to 13 years. Males predominated in $58 \%$. The length of stay ranged from 2 to 38 days. Previous hospitalization occurred in 63 patients, respiratory causes prevalent in $80.55 \%$. The irregular medical monitoring was present in 26 patients $(17.69 \%)$ and of these, nine $(34.6 \%)$ are belonging to the group who had had previous admissions.

Conclusion Of all children admitted, 115 (78.23\%) started treatment with recommended Crystalline Penicillin, and only 10 (8.7\%) required another antibiotic regimen, demonstrating the effective use of penicillin as a treatment of choice.

\section{PARACOCCIDIOIDOMYCOSIS IN CHILDREN: REPORT OF THREE CASES}

doi:10.1136/archdischild-2012-302724.0911

E Veiga, SA Nogueira, SS Cordeiro, JL Faria, A Veiga, NV Moliterno, FM Moliterno, A Siqueira, I Cordebel. Paediatrics, Faculdade de Medicina de Petrópolis, Petrópolis, Brazil

Background and Aims Paracoccidioidomycosis is the most frequent systemic mycosis in Latin America and mainly affects male adults, with a past history of working in rural areas, presenting with chronic pulmonary and mucosal lesions. However is rare in children and the clinical presentation is quite different, seemingly mostly with lymphoma or disseminated tuberculosis. The aim of this study is describe the clinical presentation, evolution and response to treatment of three cases of paracoccidioidomycosis affecting children, living in a city of Rio de Janeiro state, Brazil.

Methods Revision of the clinical charts of children who were admitted in a general pediatric ward of a teaching hospital, with confirmed diagnosis of paracoccidioidomycosis.

Results During a period time of four years, there children (two boys and one girl), were admitted with a subacute clinical picture of a generalized lymphoadenopathy (mainly cervical), fever, weighting loss, anemia, with had clinical diagnosis of lymphoma.

There was no apparent pulmonary or mucosal lesions and abdominal ultrassonography showed multiples lymphoadenopathies. Biopsy of cervical lymphonode showed Paracoccidioides brasiliensis. They were initially treated with amphotericin B and then, followed by oral itraconazol (2 cases) and ketoconazol (1 case) for one year. All had a excellent response to treatment and are being followed at the out patient clinic of infectious diseases, without relapse.

Conclusion In Latin America, Paracoccidioidomycosis should be included in the differential diagnosis of lymphoma and tuberculosis in children presenting with subacute lymphoadenopathy and biopsy looking for fungal forms is essential to establish the diagnosis.

\section{INCIDENCE OF CYTOMEGALOVIRUS(CMV) PNEUMONIA AMONG CHILDREN PRESENTING WITH SEVERE LOWER RESPIRATORY TRACT INFECTION AT DR GEORGE MUKHARI HOSPITAL}

doi:10.1136/archdischild-2012-302724.0912

${ }^{1} \mathrm{OA}$ Adewuyi, ${ }^{2} \mathrm{~S}$ Mda, ${ }^{3} \mathrm{~T}$ Kyaw. 'Department of Paediatrics and Child's Health, Medical University of Southern Africa, Medunsa/University of Limpopo; '2Department of Paediatrics and Child's Health, Medical University of Southern Africa/University of Limpopo; ${ }^{3}$ Department of Virology, University of Limpopo, Medunsa Campus, National Health Laboratory Services and Medical University of Southern Africa/University of Limpopo, Pretoria, South Africa

Background Pneumonia is a major cause of morbidity and mortality in under-five children with about 5 million deaths annually in developing countries ${ }^{1 .} \mathrm{CMV}$ is responsible for serious morbidity and mortality in immunodeficient children with pneumonia ${ }^{2}$.

Objective To determine the incidence of CMV associated pneumonia in children with severe lower respiratory tract infection (LRTI). Methods Under-5year children with severe LRTI were enrolled over a 12 months period. Severity criteria:accessory muscle use, supplemental oxygen, or assisted ventilation. Anthropometry and HIV status were recorded. Throat swabs were taken for CMV PCR and CMV serology was done. Consent and ethical approval obtained.

Results 107 children, aged 2 weeks to 46 months (mean 5.96 mths) enrolled. Incidence of laboratory confirmed CMV was 40\%(35/87); $67 \%$ among HIV-infected and $28 \%$ among HIV-uninfected $(p<0.05)$ ). Of 100 children tested for HIV infection, $30 \%$ were positive(30/100). The mean ages of HIV-infected and uninfected children were simi$\operatorname{lar}(5.83 \pm 5.77$ vs $5.99 \pm 9.43$ respectively). There was a slight difference in height-for-age Z-scores between HIV-infected $(-2.51 \pm 3.22)$ and uninfected $(-1.17 \pm 3.41)(p=0.07)$. Incidence of CMV was not associated with age or nutritional status. There were 18 deaths, $17 \%$ mortality; this was significantly higher $(p<0.01)$ among HIVinfected children (40\%) than in HIV-uninfected (9\%). Mortality was higher amongst those with positive CMV throat swabs (20\%) compared to negative CMV throat swabs (12\%), (not statistically significant). Children with a positive throat CMV were likely to receive assisted ventilation $(17 \%)$ compared to those with negative throat CMV (11.5\%); not significant ( $\mathrm{p}=0.058)$.

Conclusion Many under-5 children with severe LRTI had laboratory confirmed CMV infection. Incidence and mortality rate of CMV is higher in HIV-infected children and these patients are likely to require assisted ventilation.

\section{DETECTION OF RSV TYPES A \& B AND INFLUENZA VIRUS TYPES A \& B IN CAP BY REVERSE TRANSCRIPTION- MULTIPLEX PCR}

doi:10.1136/archdischild-2012-302724.0913

${ }^{1}$ MGE Mansour, ${ }^{2} \mathrm{~T}$ Deraz, 'S Bendary, ${ }^{1} \mathrm{~A}$ Aboelamayem. ${ }^{1}$ Ain Shams University; ${ }^{2}$ Pediatrics, Ain Shams University, Cairo, Egypt

Respiratory syncytial virus (RSV) type A and B, influenza A and B cause about $80 \%$ of viral lower respiratory tract infections. Multiplex RT-PCR has a significant advantage in that it permits simultaneous amplification of several viruses in a single reaction facilitating cost-effective diagnosis and perhaps improved clinical management. In this study, our aim was to determine the frequency of Influenza $\mathrm{A}$ and $\mathrm{B}$, and RSV types A and B among children with communityacquired pneumonia (CAP), by the use of the newly developed rapid, accurate, and pathogen-specific technique of multiplex RTPCR. This study is a cross-sectional study involving 24 children admitted to Children's Hospital of Ain Shams University due to severe lower respiratory tract infection (LRTI). Clinical and radiological assessment of all patients were performed followed by molecular analysis of both respiratory and blood samples of all 